\title{
ERRATUM
}

\section{MicroRNAs in metabolism and metabolic disorders}

Veerle Rottiers and Anders M. Näär

Nature Reviews Molecular Cell Biology 13, 239-250 (2012)

On page 250 of this article, the competing financial interests statement incorrectly stated that the authors had no competing interests. The authors' statement is reproduced below, and the article has been corrected online. The editors apologize for this omission.

Competing financial interests statement

Anders M. Näär has patents pending on miR-33a and miR-33b antisense targeting for the treatment of cardiometabolic disorders. These patents have been licensed by Santaris Pharma, Denmark. Veerle Rottiers declares no competing financial interests. 